\title{
3 Research Square

\section{Quantitative analysis of TMT phosphorylation modification to investigate the effect of verbascoside on the expression of phosphorylated protein in AD cell model}

\section{Haiyan Xing Haiyan Xing}

Inner Mongolia Medical University https://orcid.org/0000-0002-9739-504X

\section{Zhiqiang Dong Zhiqiang Dong}

First Affiliated Hospital of Baotou Medical College

Gang Li Gang Li ( $\sim 20080268 @ i m m u . e d u . c n)$

Xiaoli Du Xiaoli Du

Inner Mongolia Medical University

Lei Fan Lei Fan

Inner Mongolia Medical University

Shengnan Wang Shengnan Wang

Inner Mongolia Medical University

\section{Lulu Zhao Lulu Zhao}

Inner Mongolia Medical University

Chao Gu Chao Gu

Inner Mongolia Medical University

Yuan Gao Yuan Gao

Inner Mongolia Medical University

\section{Research article}

Keywords: verbascoside, Alzheimer's disease, phosphorylation modification, vesicle protein

Posted Date: October 27th, 2020

DOl: https://doi.org/10.21203/rs.2.24306/v3

License: (9) (1) This work is licensed under a Creative Commons Attribution 4.0 International License.

Read Full License 


\section{Abstract}

Background-The active monomer Verbascum glycosides in Cistanche tubulosa has good development prospects in terms of neuroprotection and delaying neurodegenerative diseases, and it has become one of the research hot spots. To investigate the effect of verbascoside (OC1) on the expression of phosphorylated protein in the protective effect of AD cell model by TMT labeling and phosphorylation enrichment technique and high-resolution liquid chromatography-mass spectrometry quantitative proteomics research strategy.

Methods-The normal control group, the model group $A \beta_{1-42}(10 \mu \mathrm{mol} / \mathrm{L})$ group and the $0 \mathrm{C} 1(10 \mu \mathrm{g} / \mathrm{ml})$ administration group were set. (1)protein extraction quality control. (2)TMT mark. (3)HPLC classification and modification enrichment. (4)Analysis of mass spectrometry by liquid chromatography-mass spectrometry. (5)Analysis of bioinformatics results. (6)Western Blotting was used to detect the expression levels of p-CaMKII(Thr286), p-Synapsin1(Ser603)/Synapsin1, Synaptophysin and Synaptotagmin-1 protein.

Results-The study finally identified 9020 phosphorylation sites on 3227 proteins, of which 8635 sites of 3134 proteins contained quantitative information. Screening of differential sites follows the following criteria: 1.2 times the change threshold and $\mathrm{CV}$ value $<0.1$. Based on the above data and standards, we performed a systematic bioinformatics analysis of proteins containing quantitative information sites. Western Blotting results showed that Verbascoside could promote the expression of p-CaMKII (Thr286), pSynapsin (Ser603)/Synapsin, Synaptophysin and Synaptotagmin-1 protein.

Conclusions-Verbascoside(OC1) can increase the expression of phosphorylated protein in AD cell model, which provides a basis for further study on the molecular mechanism of verbascoside promoting neurotransmitter release.

\section{Background}

Cistanches Herba is a very important TCM that was first recorded in Shen Nong Ben Cao Jing. Cistanche deserticola is a succulent stem of the genus Cistanche of the genus Cistanche of the genus $\mathrm{C}$. cerevisiae. Chemical analysis of Cistanches Herba revealed that phenylethanoid glycosides, iridoids, lignans, oligosaccharides, and polysaccharides were the main constituents. However, it has been regarded as a representative drug of Chinese medicine in the past(Ma Dongni et al.,2019). Acteoside (verbascoside), one of the main active phenylethanoid glycosides from Cistanche deserticola has demonstrated wide pharmacological activities, such as neuroprotective, immunomodulatory, anti-inflammatory, hepatoprotective and anti-oxidative, etc.(Zhang Qing et al.,2018;Yuan Yating et al., 2016;LIN Huimin et al., 2016). Previous studies have shown that acteoside can markedly reduce cerebral injury in mice induced by D-galactose (Gao et al., 2013), regulate the activity of Acetylcholine transferase(ChAT) and Acetylcholinesterase(AChE) in brain tissue, protects neurons in hippocampal CA1 region of brain tissue, improves brain tissue and immune organs index, and restores brain damage in model mice(Gao Li et al., 2014; Yang WN et al., 2014;Gao L et al., 2014; Peng Xiaoming et al., 2015; Xiong, L et al., 2015; Wu 
Hongyan et al.,2015). Furthermore, verbascoside can significantly ameliorated the cognitive dysfunction caused by $A \beta_{1-42}$ via blocking amyloid deposition, reversing cholinergic and hippocampal dopaminergic neuronal function(Hu Hang et al., 2016; Shiao YJ et al., 2017;Bai, P et al., 2013). In this way, we concluded that Cistanche tubulosa has a certain application value in the pathogenesis of learning and memory disorders such as Alzheimer's disease(AD).

Alzheimer's disease (AD), a progressive neurodegenerative disorder, is characterized by cognitive deficits. The disease is mainly characterized by memory loss,language impairment, visual spatial skills damage, slow thinking,distracted attention and affective disorder and personality changes, accompanied by the decline of social activity ability and self-life ability(Larson M E et al.,2017). Recent studies support that the neuron toxicity induced by amyloid $\beta$ peptide $(A \beta)$ (plaques) and protein tau (tangles) aggregation are closely related to AD pathogenesis. (Kenney,K et al., 2018). Insoluble $A \beta$ oligomers aggregate in extracellular plaques and were reported to lead to synaptic dysfunction, neuron toxicity and cell death. The establishment of $A D$ animal model and cell model by the infusing $A \beta_{1-42}$ into the lateral ventricle is one of the commonly used tools for studying the pathogenesis and drug treatment of $A D$ (Tian Xinhong et al., 2017).

We have previously found that verbascoside inhibits the apoptosis of neuronal cells induced by $A \beta$ during the pathogenesis of $A D$, and ameliorate the cognitive dysfunction caused by $A \beta_{1-42}$ via blocking amyloid deposition, reversing cholinergic and hippocampal dopaminergic neuronal function.( Xing Haiyan et al., 2018;Miao Xin et al., 2017;Miao Xin et al., 2017; Ju Bowei et al., 2017). It suggests that verbascoside may be an candidate neurological diseases treatment, which is associated with learning and memory dysfunction. In this study, we screened and validated the phosphorylation protein related to the protective effect of verbascoside on AD cell model, which provided a basis for further study on the molecular mechanism of verbascoside promoting neurotransmitter release.

\section{Methods}

\subsection{Materials and reagents}

PC12 cell ( purchased from the Cell Center of the Chinese Academy of Medical Sciences (H129, Beijing, China); BCA kit(183056, Thermo scientific, USA); 1640 medium (61870036هGibco, USA); FBS(1932594C》 Gibco, USA); $\beta$-amyloid (052487, Jill Biochemical Co, Ltd. China); monosialic acid IV Hexose ganglioside sodium injection(80261C10, Qilu Pharmaceutical, China); verbascoside (61276-17-3, Beijing Zhongke Quality Biotechnology Co, Ltd. China); SYN1-Specific Rabbit Polyclonal antibody(20258-1-AP, proteintech, China); Phospho-Synapsin(Ser603)Antibody

(88246, Cell Signaling, USA); Synaptotagmin-1 Rabbit mAb (14558, Cell Signaling, USA); PhosphoCaMKII(Thr286) Rabbit mAb(12716, Cell Signaling, USA); Synaptophysin Rabbit mAb(5467, Cell Signaling, USA); $\beta$-actin antibody (4970, Cell Signaling, USA); Goat Anti-Rabbit IgG(BA1054囚BOSTER), other reagents were of analytical grade; the experimental water was deionized water. 


\subsection{Experimental methods}

Through the organic combination of TMT labeling, high performance liquid chromatography grading technology, phosphorylated peptide modification and mass spectrometry-based quantitative proteomics technology, study of Phosphorylation Quantitative Omics in Samples. Its technical route is: Protein extraction- trypsin digestion- TMT/iTRAQ label- affinity enrichment- HPLC fractionation- mass spectrometry- database search- bioinformatics analysis.

\subsection{Protein extraction}

PC12 cells were cultured in high glucose DMEM containing $10 \%$ horse serum, $5 \%$ fetal bovine serum of penicillin-streptomycin and were maintained in a humidifed atmosphere containing $5 \%$ CO2. PC12 cells were divided into Control group, Model $\left.A \beta_{1-42}(10 \mu \mathrm{mol} / \mathrm{L})\right)$ group and $\mathrm{OC} 1(10 \mu \mathrm{g} / \mathrm{mL})$ group, and the cells were scraped gently after 24 hours of culture, then were sonicated three times on ice using a high intensity ultrasonic processor (Scientz) in lysis buffer (8M urea, 1\% Protease Inhibitor Cocktail). The remaining debris was removed by centrifugation at $12,000 \mathrm{~g}$ at $4^{\circ} \mathrm{C}$ for $10 \mathrm{~min}$. Finally, the supernatant was collected and the protein concentration was determined with BCA kit according to the manufacturer's instructions.

\subsection{Trypsin digestion and TMT labeling}

Dithiothreitol was added to the protein solution to a final concentration of $5 \mathrm{mM}$ and reduced at $56^{\circ} \mathrm{C}$ for $30 \mathrm{~min}$. lodoacetamide was then added to a final concentration of $11 \mathrm{mM}$ and incubated for $15 \mathrm{~min}$ at room temperature in the dark. Finally, the urea concentration of the sample is diluted to less than $2 \mathrm{M}$. Add trypsin and digest at $37{ }^{\circ} \mathrm{C}$ overnight. The tryptic digested peptides were desalted and dried in vacuo. The peptide was solubilized with 0.5M TEAB and the peptides were labeled according to the TMT kit instructions.

\subsection{HPLC classification and modification enrichment}

The tryptic peptides were fractionated into fractions by high $\mathrm{pH}$ reverse-phase HPLC using Thermo Betasil C18 column. Briefly, peptides were first separated with a gradient of $8 \%$ to $32 \%$ acetonitrile $(\mathrm{pH} 9.0)$ over 60 min into 60 fractions. After incubating the peptide in enrichment buffer, the resin was washed three times. Finally, the modified peptide was eluted with $10 \%$ ammonia water, and the eluate was collected and vacuum-dried and drained. After draining, removing salt

\subsection{Liquid Chromatography-Mass Spectrometry Analysis}

Mobile phase $A$ was an aqueous solution containing $0.1 \%$ formic acid and $2 \%$ acetonitrile; mobile phase $B$ was an aqueous solution containing $0.1 \%$ formic acid and $90 \%$ acetonitrile. Liquid phase gradient setting: 0-40 min,4\% 22\% B;40-52 min, 22\% 35\% B;52-56 min,35\% 80\% B;56-60 min,80\% B, The flow rate was maintained at $350 \mathrm{~nL} / \mathrm{min}$. The peptides were separated by ultra-high performance liquid phase system and injected into the NSI ion source for ionization and then analyzed by Q Exactive Plus mass spectrometry. The resulting MS/MS data were processed using Maxquant search engine 


\subsection{Mass spectrometry control}

As shown in the Fig.1, most of the peptides are distributed in 7-20 amino acids, which is consistent with the general rule based on trypsin enzymatic hydrolysis and HCD fragmentation. Peptides with less than 5 amino acids are too small to produce effective sequence identification. Peptides larger than 20 amino acids are not suitable for fragmentation of HCD due to their high mass and charge number. The distribution of peptide lengths identified by mass spectrometry meets quality control requirements.

Fig.1 Mass spectrometry identified the length distribution of peptides and identification of protein corresponding modification site distribution

\subsection{Bioinformatics analysis method}

\subsubsection{Protein annotation method}

Gene Ontology analysis: Gene Ontology analysis, or GO analysis, is a bioinformatics analysis method that can link information between genes and proteins to provide statistical information. Gene Ontology analysis mainly includes three aspects: 1 . Cell composition 2 . Molecular function 3 . Biological process.

KEGG pathway annotation: KEGG is able to integrate currently known protein interaction network information. We used the KEGG pathway database to annotate the protein pathway to match the protein to the corresponding pathway in the database.

Subcellular localization: Proteins in eukaryotic tissue cells are located in detail on various elements within the cell, depending on the membrane structure with which they bind. We used a software for predicting subcellular localization wolfpsort to perform subcellular localization annotation of the submitted protein.

\subsubsection{Functional Enrichment}

\subsubsection{Enrichment of Gene Ontology analysis}

Proteins were classified by GO annotation into three categories: biological process, cellular compartment and molecular function.For each category, a two-tailed Fisher's exact test was employed to test the enrichment of the differentially modified protein against all identified proteins. The GO with a corrected pvalue less than 0.05 is considered significantly.

\subsubsection{Enrichment of pathway analysis}

Encyclopedia of Genes and Genomes(KEGG) database was used to identify enriched pathways by a twotailed Fisher's exact test to test the enrichment of the differentially modified protein against all identified proteins. The pathway with a corrected $p$-value less than 0.05 was considered significant. These pathways were classified into hierarchical categories according to the KEGG website. 


\subsubsection{Enrichment of protein domain analysis}

For each category proteins, InterPro(a resource that provides functional analysis of protein sequences by classifying them into families and predicting the presence of domains and important sites)database was researched and a two-tailed Fisher's exact test was employed to test the enrichment of the differentially modified protein against all identified proteins. Protein domains with a corrected $p$-value less than 0.05 were considered significant.

\subsubsection{Cluster analysis based on protein function enrichment}

Cluster analysis based on functional enrichment of differentially modified proteins to study their potential associations and differences in the KEGG pathway. We first collect functional classification information enriched for the protein used and the corresponding enriched P-value values, and then screen out functional classifications that are significantly enriched in at least one protein group. The P-value data matrix obtained by the screening is first subjected to logarithmic transformation with -log10, and then the transformed data matrix is subjected to $Z$ transformation for each function classification. Finally, the data set obtained after $Z$ transformation is analyzed by hierarchical clustering. The clustering relationship is visualized using the heat map drawn by the function heatmap. 2 in the $\mathrm{R}$ language package gplots.

\subsubsection{Analysis of modified site motifs}

Software MoMo, the motif-x algorithm was used to analyze the motif features of the modified sites. Among them, 10 identified phosphorylations from upstream and downstream of each identified modification site were peptide fragments consisting of 6 amino acids from upstream to downstream. The analysis background was 10 upstream and downstream of all potential modification sites in the species.

\subsection{Western blotting}

PC12 cells were harvested and washed twice with PBS solution after drug treatment. cells were lysed using RIPA Lysis Buffer. The lysates were collected by scraping from the plates, and then they were centrifuged. Total protein samples were denatured and loaded on a SDS-polyacrylamide gel for electrophoresis, and then transferred onto PVDF transfer membranes. Membranes were blocked at room temperature for $2 \mathrm{~h}$ with blocking solution. Membranes were incubated overnight at $4 \circ \mathrm{C}$ with primary antibodies:p-CaMKII(Thr286)(1:1000),Synapsin(1:2000),p-Synapsin(Ser603)(1:1000), Synaptophysin(1:1000), Synaptotagmin-1(1:1000) and $\beta$-Actin(1:1000)dilution in blocking solution. After three washings, the membranes were incubated with secondary horseradish peroxidase (HRP)-conjugated goat anti-rabbit immunoglobulin $\mathrm{G}$. Then the proteins were detected using an enhanced chemiluminescence detection kit. The images were obtained using a UVP ChemStudio gel imaging system (Analytik Jena AG)『and the band intensities were quantified with ImageJ software.

\subsection{Statistics}

Results are shown as the mean \pm SD. Diferences between two experimental conditions were evaluated with one-way ANOVA. The data were analyzed using SPSS 22.0 software, and a value of $p<0.05$ was 
considered to indicate a statistically significant difference.

\section{Results}

\subsection{Overview of modification identification}

In this project, a total of 53460 secondary spectra were obtained by mass spectrometry. After the mass spectrometry secondary spectrum was searched by protein theory data, the available effective spectrum number was 19226, the spectrum utilization rate was $36.0 \%$, and 9972 peptides were identified by spectral analysis. 8705 phosphorylated peptides were identified. We identified a total of 11325 phosphorylation sites on 3448 proteins, of which 10118 sites on 3309 proteins have quantitative information (Table 1, Fig. 2).

Table 1 Basic statistical table of mass spectrometry data results

\begin{tabular}{cccccccc}
\hline $\begin{array}{c}\text { Total } \\
\text { spectrum }\end{array}$ & $\begin{array}{c}\text { Matched } \\
\text { spectrum }\end{array}$ & Peptides & $\begin{array}{c}\text { Modified } \\
\text { peptides }\end{array}$ & $\begin{array}{c}\text { Identified } \\
\text { proteins }\end{array}$ & $\begin{array}{c}\text { Quantifiable } \\
\text { proteins }\end{array}$ & $\begin{array}{c}\text { Identified } \\
\text { sites }\end{array}$ & $\begin{array}{c}\text { Quantifiable } \\
\text { sites }\end{array}$ \\
\hline 53460.0 & 19226 & 9972.0 & 8705.0 & 3448 & 3309 & 11325 & 10118 \\
& $(36.0 \%)$ & & & $(3227)$ & $(3134)$ & $(9020)$ & $(8635)$ \\
& & & & & & & \\
\hline
\end{tabular}

\subsection{Differential modification analysis of OC1 on phosphorylated protein in AD cell model}

The quantified value of the modified peptide corresponding to each sample was determined by mass spectrometry quantitative analysis. For each repeated experiment, the ratio of the quantitative values between the two different samples was taken as the comparative group difference expression(Ratio). Since this project has performed two repeated experiments, for each comparison group,we take the average of the two repeated ratio values as the ratio value of the comparison group, and the coefficient of variation $\mathrm{CV}$ of the two repeated Ratio values as the comparison group. When CV-value less than 0.1 , the difference expression amount changes by more than 1.2 as a significantly up-regulated change threshold, and less than $1 / 1.2$ as a significantly down-regulated change threshold. The summary data of all differentially expressed proteins. From the results, it can be seen that compared with the control group, $A \beta_{1-42}(10 \mu \mathrm{mol} / \mathrm{L})$ treated cells for 24 hours, the expression of 3083 sites at 1,886 proteins changed, of which 789 sites were up-regulated and 2294 sites Site down-regulation; Compared with the Model(A $\left.\beta_{1-42}\right)$ group, the expression of 991 loci of 740 proteins changed after $0 \mathrm{C} 1(10 \mu \mathrm{g} / \mathrm{mL})$ administration, including 341 loci up-regulation and 650 loci down-regulation, as shown in (Table 2 and Fig.3 ).

Table2 Modification level difference modification statistics 


\begin{tabular}{cccc}
\hline Compared sample name & Type & Up-regulated & Down-regulated \\
\hline Model/Control & Sites & 789.0 & 2294.0 \\
& Proteins & 570.0 & 1316.0 \\
OC1/Control & Sites & 858.0 & 3430.0 \\
& Proteins & 589.0 & 1797.0 \\
OC1/Model & Sites & 341.0 & 650.0 \\
& Proteins & 261.0 & 479.0 \\
\hline
\end{tabular}

\subsection{Sample repeatability test}

For biological replicates or technically replicated samples, we examined whether the quantitative results of biological replicates or technical replicates were statistically consistent. Here we use the relative standard deviation(RSD) statistical analysis method to evaluate the quantitative repeatability of the modification. As shown in Fig.4, the overall RSD value is small and the quantitative repeatability is good.

\subsection{Functional enrichment and cluster analysis of differentially modified proteins in AD cell model after OC1 administration}

Based on the identification of all the proteins containing the modified site proteins and the screening of the proteins corresponding to the differentially modified sites, we performed enrichment analysis and cluster analysis to detect whether the differential modifications have significant enrichment trend in some functional types.

\subsubsection{OC1 increases the expression of differentially modified proteins associated with calcium channels}

In order to detect whether the differential modification has a significant enrichment trend in certain functional types, we performed GO classification, KEGG pathway, and protein domain enrichment analysis on all differentially modified proteins identified by the screening. For the $p$-value obtained from the enrichment test,the functional classification and pathway of significant enrichment of differentially modified proteins $(P<0.05)$ were demonstrated by means of a bubble chart. In Fig.5, the vertical axis is the functional classification or pathway, and the horizontal axis is the log2 converted value of the ratio of the percentage of the differentially modified protein in the functional type compared to the identified protein. It can be seen from the figure that through the three-level enrichment analysis, we have obtained differentially modified proteins related to calcium channel activation after OC1 administration(Fig.5)

\subsubsection{OC1 increases the expression of differentially modified protein p-CaMKロ (Thr286) related to calcium signaling pathway}


After enriching the differentially modified proteins in different comparison groups, we performed KEGG cluster analysis on them to find the correlation of the function of the differentially modified proteins after $\mathrm{OC} 1$ administration. According to the enrichment test $\mathrm{P}$ value obtained from the enrichment analysis, the related functions in different groups are brought together to draw a heat map using a hierarchical clustering method. The horizontal direction of the heat map represents different comparison groups, and the vertical direction describes the functions related to differential modification and enrichment. Different color blocks indicate the degree of enrichment, red means that the degree of enrichment is strong, and blue means that the degree of enrichment is weak.Compared with the Model $\left(A \beta_{1-42}\right)$ group, differentially modified proteins were enriched to the $\mathrm{Ca}^{2+}$ signaling pathway (see Fig. $6 \mathrm{~A}$ ) after $\mathrm{OC} 1$ administration. In this pathway we found that the expression of the protein p-CaMK\(Thr286) was up-regulated after OC1 administration (see Figure 6B). CaMKII is one of the most abundant kinases in neurons, comprising $1-2 \%$ of the total protein concentration. This multifunctional kinase is a major mediator of calcium signaling in neurons, which can further affect the substrate Synapsin1(Syn1) (Ser603). Participate in the remodeling of synapses, mediate the release of transmitters and participate in learning and memory.

\subsection{CaMKII(Thr286) and Syn1(Ser603)Protein modification motif analysis}

The protein motif analysis calculates the regularity of the amino acid sequence in the phosphorylation modification site by counting the regularity of the amino acid sequence before and after all phosphorylation sites in the sample. This analysis revealed the sequence characteristics of the modified sites of the target proteins p-CaMKII(Thr286) and p-Syn1(Ser603), then provide evidence for subsequent discovery of related proteins(Table 3 ).

Table 3 CaMKII(Thr286) and Syn1(Ser603) Modification Site Sequences

\begin{tabular}{cccccc}
\hline $\begin{array}{c}\text { Protein } \\
\text { accession }\end{array}$ & Position & $\begin{array}{c}\text { Amino } \\
\text { acid }\end{array}$ & $\begin{array}{c}\text { Gene } \\
\text { name }\end{array}$ & $\begin{array}{c}\text { Subcellular } \\
\text { localization }\end{array}$ & Motif \\
\hline F1LNI8 & 286 & Thr & CaMKIIb & cytoplasm & xxxRxx_S_xxxxxx \\
P15791 & 286 & Thr & CaMKIId & cytoplasm & xxxxxx_S_Dxxxxx \\
P09951 & 603 & Ser & Syn1 & nucleus & xxxRxx_S_xxxxxx \\
\hline
\end{tabular}

\subsection{GO secondary annotation classification}

To understand the modified proteins identified and quantified in the data, we have detailed comments on the functions and characteristics of these proteins. Gene Ontology $(\mathrm{GO})$ is a gene theory that expresses various properties of genes and gene products. $\mathrm{GO}$ annotations are divided into three broad categories: Biological Process, Cellular Component,and Molecular Function,which explain the biological effects of proteins from different perspectives. We calculated the distribution of the differentially modified loci corresponding proteins p-CaMKII(Thr286) and p-Syn1(Ser603) in the GO secondary annotation.As shown in Fig.7, p-CaMKII(Thr286) and p-Syn1(Ser603) are mainly located in the cell junction and synapse(Fig.7) 


\subsection{Effects of OC1 on A 3 -induced changes in p-CaMKロ (Thr286), p-Synapsin1(Ser603)/ Synapsin1, Synaptophysin, and Synaptotagmin-1}

Our results demonstrate that $\mathrm{OC} 1$ protects $\mathrm{PC} 12$ cells from $\mathrm{A} \beta_{1-42}$-induced cytotoxicity and improves cell viability. Monosialyltetrahexose gangliosides are a class of glycosphingolipids that contain sialic acid widely on the cell membranes of vertebrates and are closely related to the formation, elongation and

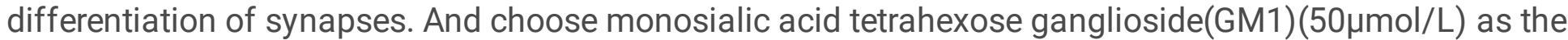
positive drug(Wei Guan et al., 2017; Hu Xufeng et al., 2017). Western blot assay applied to detect the effect of OC1 on p-CaMKII(Thr286) and vesicle-related proteins in AD cell model. The results showed that compared with the control group, the expression of $\mathrm{p}$-CaMK囚(Thr286) and vesicle proteins $\mathrm{p}$ Synapsin1(Ser603)/Synapsin1, Synaptophysin,and Synaptotagmin-1 in the A 1 1-42 group were significantly reduced $(\mathrm{P}<0.05)$; on the contrary, the expression of $\mathrm{p}$-Synapsin $1(\mathrm{Ser603}) /$ Synapsin 1 and Synaptophysin increased by about $20.59 \%$ and $31.70 \%$, respectively, in the $0 \mathrm{C} 1(2 \mu \mathrm{g} / \mathrm{mL})$ group, compared with the Aß1-42 group, Moreover, the expressions of $p$-CaMK囚(Thr286) and vesicular proteins $p$ Synapsin1(Ser603)/Synapsin1, Synaptophysin, and Synaptotagmin-1 increased by approximately $30.67 \%, 48.53 \%, 139.5 \%$, and $84.48 \%(P<0.05)$; Compared with the $A \beta_{1-42}$ group, the expression of $p$ CaMK区(Thr286), p-Synapsin1(Ser603)/Synapsin1, Synaptophysin, and Synaptotagmin- 1 were increased by approximately $32.00 \%, 38.24 \%, 102.3 \%$, and $91.37 \%(P<0.05)$, show in Fig. 8 .

\section{Discussion}

$A \beta_{1-42}$ is a metabolite of Amyloid precursor protein(APP). Recent studies have indicated that the nonfibrillar soluble oligomeric form of amyloid $\beta$ protein (sA $\beta$ ) rather than insoluble amyloid fibrils or plaques is the cause of the synaptic dysfunction and cognitive defects associated with AD. It has been reported that autophagy plays an important role in the generation and metabolism of $A \beta$, and thus its malfunction may lead to the progress of $A D$ (Toneff T et al., 2013). Therefore, $A \beta$-induced apoptosis in PC12 cells was a reliable cellular toxicity model for $A D$ related studies in vitro. In our experiment, the $10 \mu \mathrm{mol} / \mathrm{L} A \beta_{1-42}$ induced cell model was established, and it was found that pre-treatment with $10 \mu \mathrm{g} / \mathrm{mL}$ of OC1 could effectively improve cell viability and reduce the change of cell morphology.

Protein phosphorylation is one of the most important posttranslational modifications in living cells and is involved in the regulation of a large number of biological processes, such as cell cycle, signal transduction, differentiation, proliferation, metabolism, and autophagy. Whether it is normal physiological processes or cytotoxic protein aggregation in neurodegenerative diseases, it is related to posttranslational modification of proteins. Phosphorylation is the most widely studied post-translational modification of proteins, besides of phosphorylation of serine, threonine, and tyrosine. Phosphorylation is involved in almost all cellular processes and plays a critical role in the release of neurotransmitters. Advances in proteomic technology with a rapid development of liquid chromatographytandem mass spectrometry (LC/MS-MS) have provided a great opportunity to study phosphorylation events in detail. In this experiment, TMT labeling and phosphorylation modification enrichment technology and highresolution liquid chromatography-mass spectrometry combined with quantitative proteomics were used to 
study the effect of verbascoside on A -induced Rat adrenal medulla pheochromocytoma cells(PC12) cellrelated vesicle protein expression(Zhiqiang W et al., 2018;Erickson B K et al., 2014;Weekes $\mathrm{M}$ et al., 2014). In the present study, $\mathrm{OC} 1$ can increase the expression of $\mathrm{p}$-CaMKII(Thr286) protein in AD cell model.

$\mathrm{AD}$ patients and is primarily due to disrupting synaptic plasticity, $\mathrm{Ca}^{2+}$ homeostasis and signaling pathways such as $\mathrm{Ca}^{2+} /$ calmodulin-dependent protein kinase kinase. As an important second messenger, $\mathrm{Ca}^{2+}$ enrolls in various physiological and biochemical processes in the body. The $\mathrm{Ca}^{2+} / \mathrm{CaM}-\mathrm{CaMKII}$ signaling pathway plays an important role in the formation and maintenance of learning and memory in the central nervous system. CaM is an important signaling molecule in the cell.It combines with $\mathrm{Ca}^{2+}$ to form a $\mathrm{Ca}^{2+} / \mathrm{CaM}$ complex. It activates downstream CaMKII to participate in various physiological effects such as learning and memory(Sun Jingran et al., 2018). CaMKII as a multifunctional serine/threonine protein kinase. The unique properties, keypositions, and complex regulation of CaMKII allow it to participate in important synaptic functions, including the synthesis and release of neurotransmitters. (MIN D Y et al., 2012). $\mathrm{Ca}^{2+}$-CaM induces the conformational change of CaMKII, and activated CaMKII can activate the intranuclear transcription mechanism of neurons, thereby regulating the synthesis of substrates downstream and downstream of neurons( Liyan Z et al., 2018).

Mo04020 Calcium signaling pathway was obtained from KEGG signal pathway enrichment and clustering. Calcium enters cells and activates CaMKII protein. Synaptic vesicles are a very small and highly specialized organelle whose function is known to store and release neurotransmitters. Synapsinl is a nerve terminal-specific phosphoprotein involved in synaptic transmission, neural development, and neurite outgrowth(Zhu L et al., 2018;Liu Xiao et al., 2017). After being activated by CaMKII, Phosphorylated Synapsin I causes SVs to dissociate from the actin cytoskeleton(Chen X et al., 2018). Synaptophysin is a synaptic vesicle glycoprotein that is widely expressed on presynaptic membranes and strongly associated with synaptic plasticity. Elevated Synaptophysin levels have a positive effect on consolidation of learning and memory after injury. During the release of neurotransmitters, it will move the vesicles to the plasma membrane and promote the formation of SNARE complexes(Südhof,ThomasC,2013). Calcium-binding protein Synaptagmin is a protein located in the synaptic vesicle membrane.After being induced by $\mathrm{Ca}^{2+}$, it can be inserted into the presynaptic membrane or act on the fusion pore(Ciani L et al., 2015;Sudhof, T. C., 2015;Jiong Tang et al., 2016), after which, under the combined influence of the SNARE complex and $\mathrm{Ca}^{2+}, \mathrm{SV}$ s anchor and fuse to the synaptic membrane, enabling the release of neurotransmitters into the synaptic cleft. The experimental Western Blot results showed that OC1 can increase the expression of p-CaMKII(Thr286),p-Synapsin1(Ser603), synaptophysin and synaptotagmin-1 in PC12 cells after $A \beta_{1-42}$ intervention. These results indicated that $\mathrm{OC} 1$ can affect the release of neurotransmitters by increasing the expression of synaptic vesicle-related proteins.

\section{Conclusion}

Through phosphorylation modification enrichment technology and quantitative proteomics analysis results, we can conclude safely that verbascoside can increase the expression of CaMKII protein threonine 
286 phosphorylation by affecting the amount of calcium ions, which in turn triggers downstream synaptic vesicle correlation. In the future, we will continue to carry out in-vivo experimental verification to further improve the research on the role of verbascoside on the nervous system.

\section{Abbreviations}

Rat adrenal medulla pheochromocytoma cells (PC12 cells)

Verbascoside(OC1)

$\beta$-amyloid (AB)

Alzheimer 's disease (AD)

Monosialic acid tetrahexose ganglioside (GM1)

Gene Ontology (GO)

\section{Declarations}

\section{Ethics approval and consent to participate}

Not applicable

Consent for publication

Not applicable

\section{Availability of data and materials}

The datasets generated and analysed during the current study are not publicly available due the research group will continue to conduct in-depth data mining and verification, but are available from the corresponding author on reasonable request.

\section{Competing interests}

No scramble for interest in this section

\section{Funding}

This study was supported by Inner Mongolia Natural Science Foundation Project (2018MS08026) and State Key Laboratory of Natural Pharmaceutical Active Substances and Functions (GTZK201710). The funding bodies played no role in the design of the study and collection, analysis, and interpretation of data and in writing the manuscript.

\section{Authors' contributions}


GL and HX participated in designing, data collection, analysis, interpretation, and manuscript writing; SW, LZ, CG and YG participated in data analysis, results interpretation; ZD, XD and LF guided the conduct of the experiment and the revision and polishing of the manuscript. All authors have read and approved the final version of the manuscript.

\section{Acknowledgements}

Thanks to Dr. GL and the students in the laboratory for their free help in this experimental research

\section{References}

Ma Dongni, Li Yun, Yang Xiujuan, Dai Hairong, Zhang Lijun, Fan Qin.Research progress on harvest processing and processing methods of Cistanche cistantros [J] .China Pharmacy, 2019,30 (06): 839-841.

Zhang Qing, Li Yazhen, Sun Zhihui, Xia Meiru. Research Progress on Antioxidant Activity of Active Substances in Cistanche [J]. Agricultural Products Processing, 2018 (21): 60-62.

Yuan Yating, Hu Guili, Ding Peng, Wang Yushi, Zhang Shirui, He Xi. Physiological functions of mullein and its application in animal production [J]. Journal of Animal Nutrition, 2016, 28 (12): 3777-3783.

LIN Huimin, DUAN Weibing, SHAO Rui, HAN Lifeng, ZHU Yan, GAO Xiumei, and Wang Yan. Mullein promotes neural stem cell proliferation in adult mice by activating the PI3K / AKT pathway [J] .Chinese Pharmacological Bulletin, 2016,32 (06): 836-840 .

Gao Li, Peng Xiaoming, Huo Shixia, Lin Juan, Yan Ming, Zhang Fuchun. The role of mullein in improving D-galactose-induced subacute aging brain damage in mice [J]. Chinese Traditional and Herbal Drugs, 2014, 45 (01): 81- 85.

Yang WN, Hu XD, Han H, et al. The effects of valsartan on cognitive deficits induced by aluminum trichloride and d-galactose in mice. Neurol Res .2014.36: 651-658.

Gao, L.; Peng, X.; Huo, S.; He, Y.; Yan, M. Acteoside enhances expression of neurotrophin-3 in brain tissues of subacute aging mice induced by D-galactose combined with aluminum trichloride. Chin. J. Cell. Mol. Immunol. 2014, 30, 1022-1025

Xiao-Ming Peng, Li Gao, Shi-Xia Huo,囚. The Mechanism of Memory Enhancement of Acteoside (Verbascoside) in the Senescent Mouse Model Induced by a Combination of d -gal and $\mathrm{AlCl} 3[\mathrm{~J}]$. Phytotherapy Research Ptr, 2015, 29(8):1137-1144.

Xiong, L.; Mao, S.; Lu, B.; Yang, J.; Zhou, F.; Hu, Y.; Jiang, Y.; Shen, C.; Zhao, Y. Osmanthus fragrans ffflower extract and acteoside protect against D-galactose-induced aging in an ICR mouse model. J. Med. Food $2016,19,54-61$. 
Wu Hongyan, Li Hailong, Gu Jing, Lan Meihua, Wang Huping, Che Min, Deng Jiannan.Effect of Hei Xiaoyao Powder on $A \beta_{25-35}$ Induced Neurotransmitters and Hippocampal Pathological Changes in AD Rats [J] .China Journal of Gerontology, 2015, 35 (16): 4417-4420.

Hu Hang. Research on the protective effect and mechanism of mullein on $A \beta_{1-42}$ injured neurons [J]. Journal of Liaoning University of Traditional Chinese Medicine, 2016, 18 (10): 34-37.

Shiao YJ, Su MH, Lin HC, Wu CR. Acteoside and Isoacteoside Protect Amyloid $\beta$ Peptide Induced Cytotoxicity, Cognitive Deficit and Neurochemical Disturbances In Vitro and In Vivo. Int J Mol Sci. 2017;18(4):895. Published 2017 Apr 24.

Bai, P.; Peng, X.M.; Gao, L.; Huo, S.X.; Zhao, P.P.; Yan, M. Study on protective effect of acteoside on cellular model of Alzheimer's disease induced by okadaic acid. China J. Chin. Mater. Med. 2013, 38, 1323-1326.

Larson M E , Greimel S J , Amar F, et al. Selective lowering of synapsins induced by oligomeric asynuclein exacerbates memory deficits[J]. Proceedings of the National Academy of Sciences, 2017, 114(23):E4648.

Kenney, K., lacono, D., Edlow, B., et al. Dementia After Moderate-Severe Traumatic Brain Injury:Coexistence of Multiple Proteinopathies[J]. J Neuropathol Exp Neurol, 2018, 77 (1) :50-63.

Tian Xinhong, Hao Li, You Yanwen, Xu Yuying, Yu Shiqi. Effect of Ginkgolide B on the Expression of Synapsin-1, Beclin1 and LC3 in the Hippocampus of Alzheimer's Disease Rats [J]. China Journal of Basic Medicine in Traditional Chinese Medicine, 2017, 23 (12): 1701-1704 + 1759.

Xing Haiyan, Miao Xin, Zhang Xiaofei, Liu Dandan, Ma Zixing, Li Gang. BDNF acetylation participates in the protective effect of mullein on $A \beta_{-}$(25-35) -induced PC12 cell damage [J]. China Journal of New Drugs, 2018, 27 (16): 1910-1917.

Miao Xin, Zhang Hong, Wu Yan, Liu Dandan, Zhang Xiaofei, Li Gang. Protective effects of Cistanche deserticola glycosides on D-galactose-induced PC12 nerve cell injury [J]. China Pharmaceutical Journal, 2017, 52 (23): $2071-2078$.

Miao Xin, Zhang Hong, Bu Ren, Wu Yan, Liu Dandan, Zhang Xiaofei, Zhao Guojun, Li Gang. UPLC / Q Exactive MS Detection of Cistanche Polysaccharides Promoting Release of Neurotransmitters from PC12 Cells [J]. Journal of Pharmaceutical Analysis, 2017, 37 (09): 1667-1674.

Ju Bowei, Yang Jianhua, Yan Yao, Cao Dandan, Xiang Xueyong, Hu Junping. Protective effect of cistanche total glycosides on $A \beta_{1-42}$-induced PC12 cell damage[J].Journal of Xinjiang Medical University, 2017 , 40 (05): 634-637.

Wei Guan, Xin Yanrui, Han Huiru. Clinical efficacy and safety of edaravone combined with gangliosides in the treatment of elderly patients with acute cerebral infarction [J]. Primary Medical Forum, 2018, 22 (16): 
Hu Xufeng, Fang Chao, Jiang Yunyun.Effects of gangliosides on the expression of Caspase-3 in rats with acute spinal cord injury and its inhibition of neuronal apoptosis [J] .Journal of Qiqihar Medical College, 2018,39 (15): 1737-1740.

Toneff $T$, Funkelstein $L$, Mosier $C$, et al. Beta-amyloid peptides undergo regulated co-secretion with neuropeptide and catecholamine neurotransmitters[J]. Peptides, 2013, 46(Complete):126-135.

Zhiqiang W, Jing M , Chika M , et al. Quantitative phosphoproteomic analysis of the molecular substrates of sleep need[J]. Nature, 2018.

Erickson B K, Jedrychowski M P, Mcalister G C , et al. Evaluating Multiplexed Quantitative Phosphopeptide Analysis on a Hybrid Quadrupole Mass Filter/Linear lon Trap/Orbitrap Mass Spectrometer[J]. Analytical Chemistry, 2014, 87(2).

Weekes M , Tomasec P, Huttlin E , et al. Quantitative Temporal Viromics: An Approach to Investigate HostPathogen Interaction[J]. Cell, 2014, 157(6):1460-1472.

Sun Jingran, Li Chaonan, Li Li, Liu Zhongwen, Fang Yanjun.Effect of TDCPP exposure on CaMKII and MAPK signal pathway in PC12 cells [J] .Medical Medicine, 2018,42 (04): 266-270.

MIN D Y, MAO X Y, WU K C, et al. Donepezil attenuates hippocampal neuronal damage and cognitive deficits after global cerebral ischemia in gerbils[J]. Neuroscience Letters,2012,510(1)『29-33.

Liyan Z , Yonghai Z , Fan Y, et al. Effects of intrathecal bupivacaine on the NR2B/CaMKIla/CREB signaling pathway in the rat lumbar spinal cord[J]. Molecular Medicine Reports, 2018.

Zhu $L$, Tang $T$, Fan $R$, et al. Xuefu Zhuyu decoction improves neurological dysfunction by increasing synapsin expression after traumatic brain injury[J]. Neural Regeneration Research, 2018, 13(8):1417.

Liu Xiao, Wang Hu. Effect of Danshen injection on neural stem cell transplantation for craniocerebral injury [J]. China Tissue Engineering Research, 2017, 21 (29): 4709-4715.

Chen $\mathrm{X}$,Wang $\mathrm{X}$,Yang $\mathrm{Y}$, et al.Schwann cells protect against CaMKII- and PKA-dependent Acrylamideinduced Synapsin I phosphorylation[J].Brain Res. 2018,7

Südhof, ThomasC. Neurotransmitter Release: The Last Millisecond in the Life of a Synaptic Vesicle[J]. Neuron, 2013, 80(3):675-690.

Ciani L, Marzo A, Boyle K, et al. Wnt signalling tunes neurotransmitter release by directly targeting Synaptotagmin-1[J]. Nature Communications, 2015, 6:8302.

Sudhof, T. C . Calcium Control of Neurotransmitter Release[J]. Cold Spring Harbor Perspectives in Biology, 2012, 4(1):0-0. 
Jiong Tang, Anton Maximov, Ok-Ho Shin.et al. A Complexin/Synaptotagmin 1 Switch Controls Fast Synaptic Vesicle Exocytosis[J]. 126(6):0-1187.

\section{Figures}
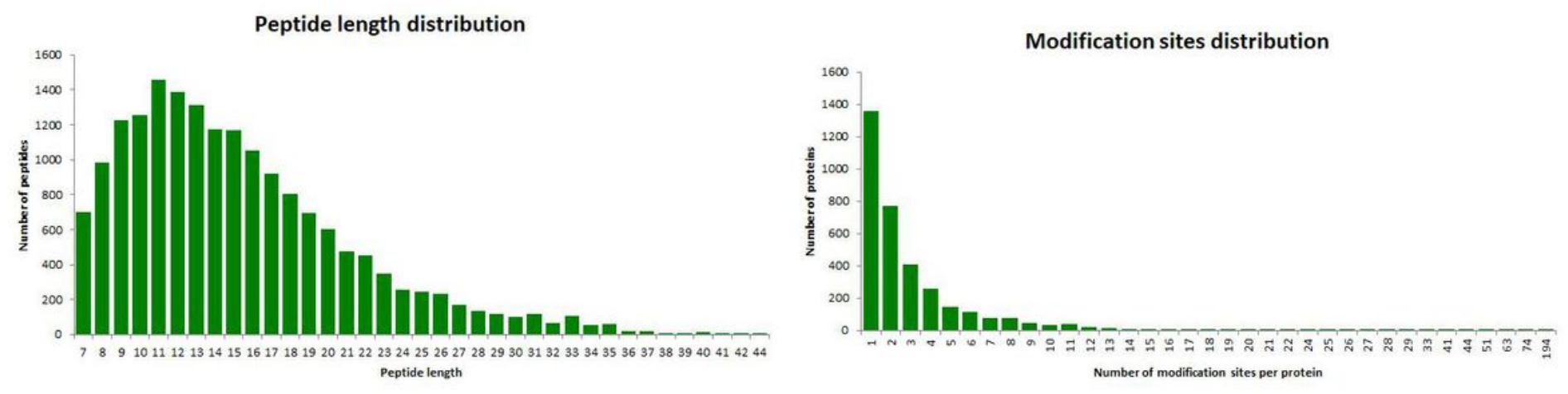

\section{Figure 1}

Mass spectrometry identified the length distribution of peptides and identification of protein corresponding modification site distribution 


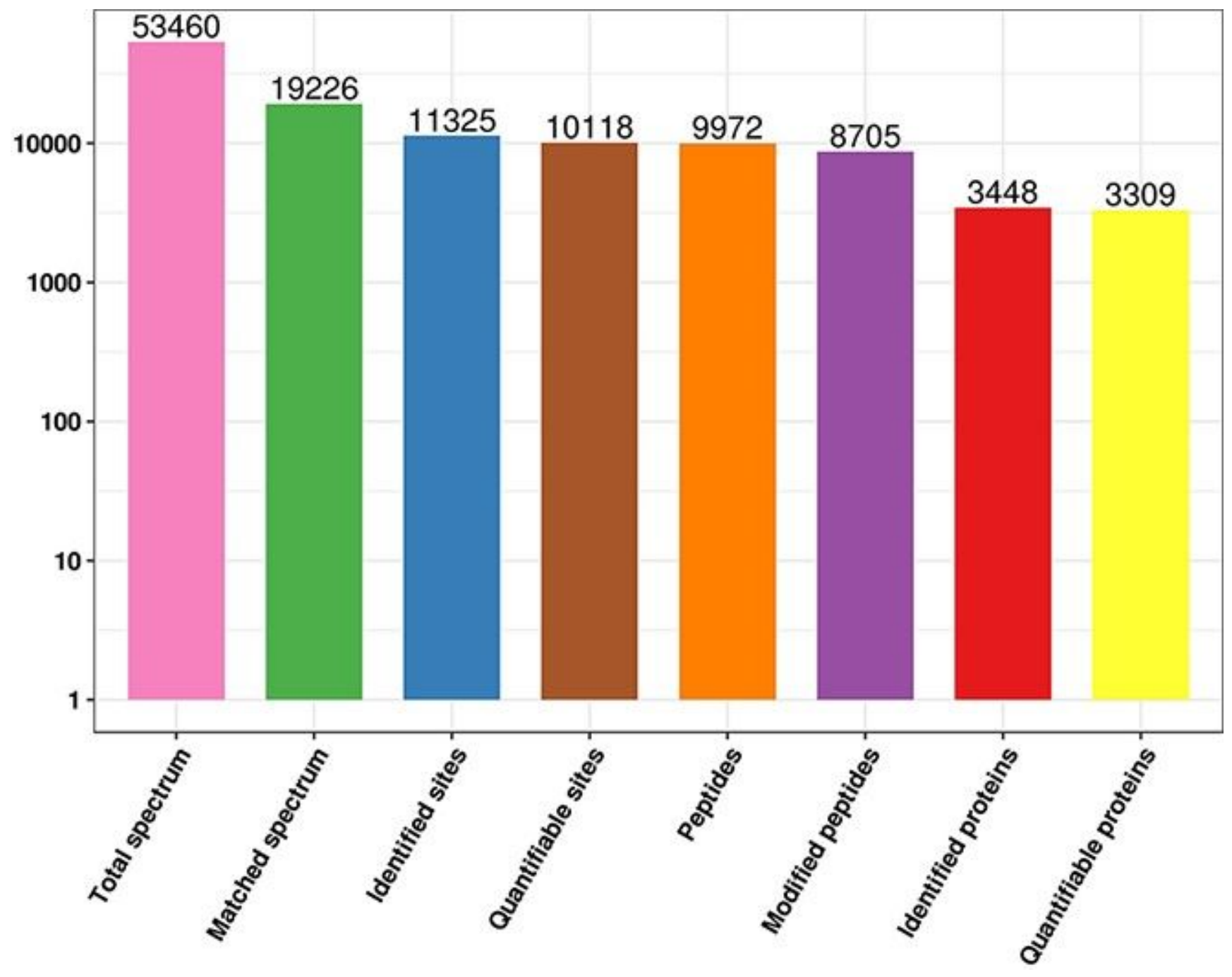

Figure 2

Basic statistics of mass spectrometry data results 


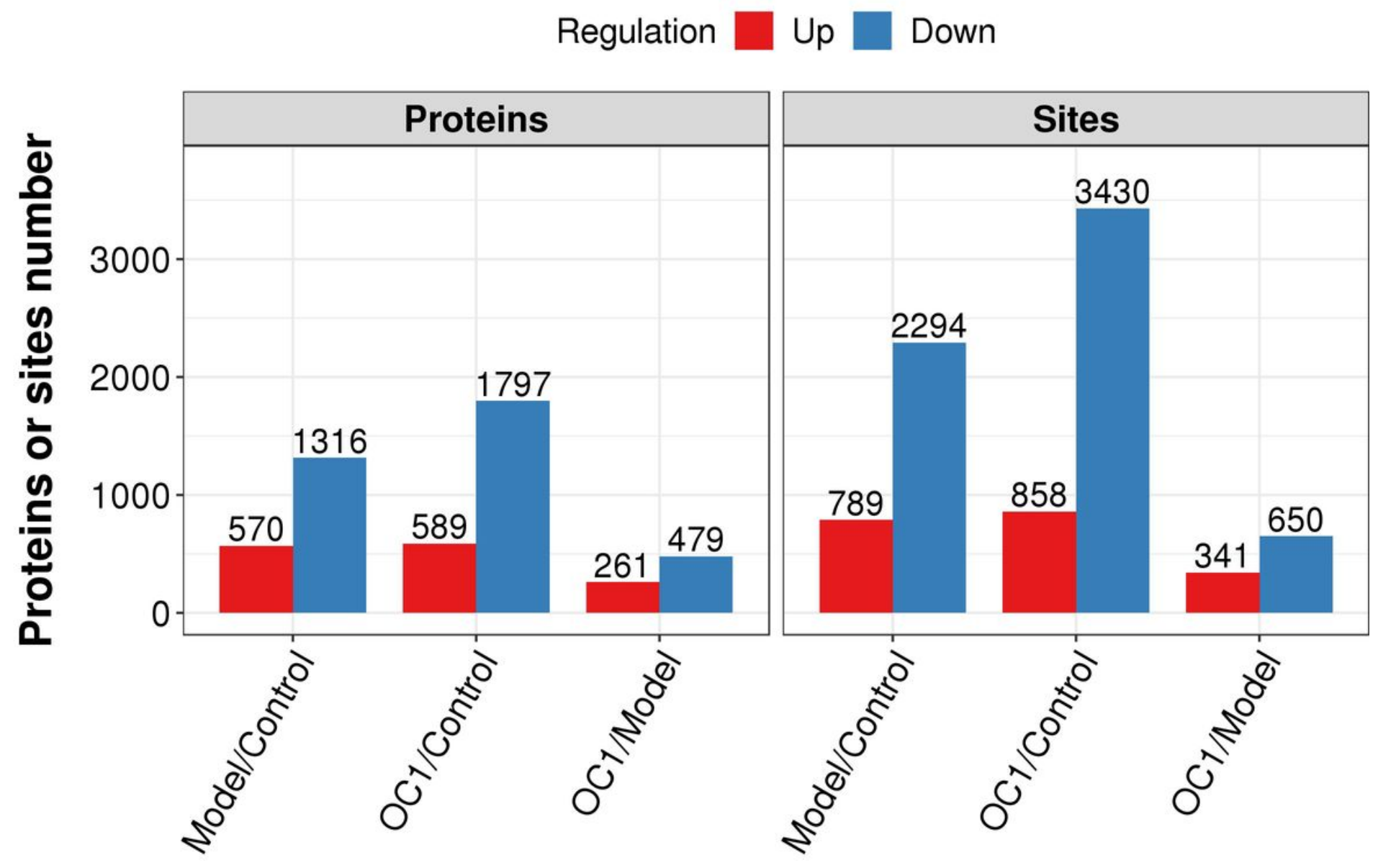

Figure 3

Bar graph of the number distribution of differentially modified proteins and modified sites in different comparison groups. 


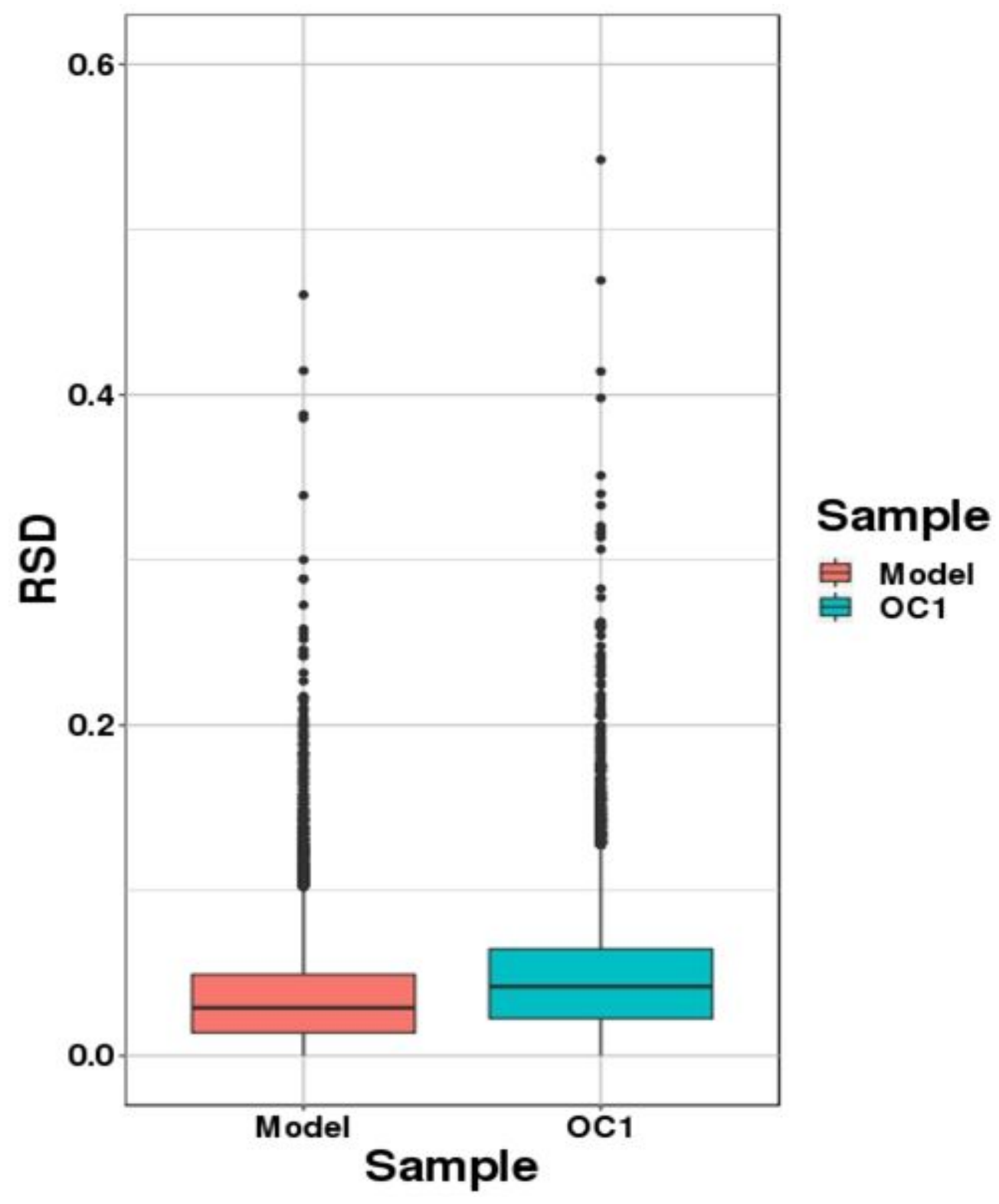

Figure 4

Repeated sample-to-sample modification quantitative RSD distribution box line diagram 

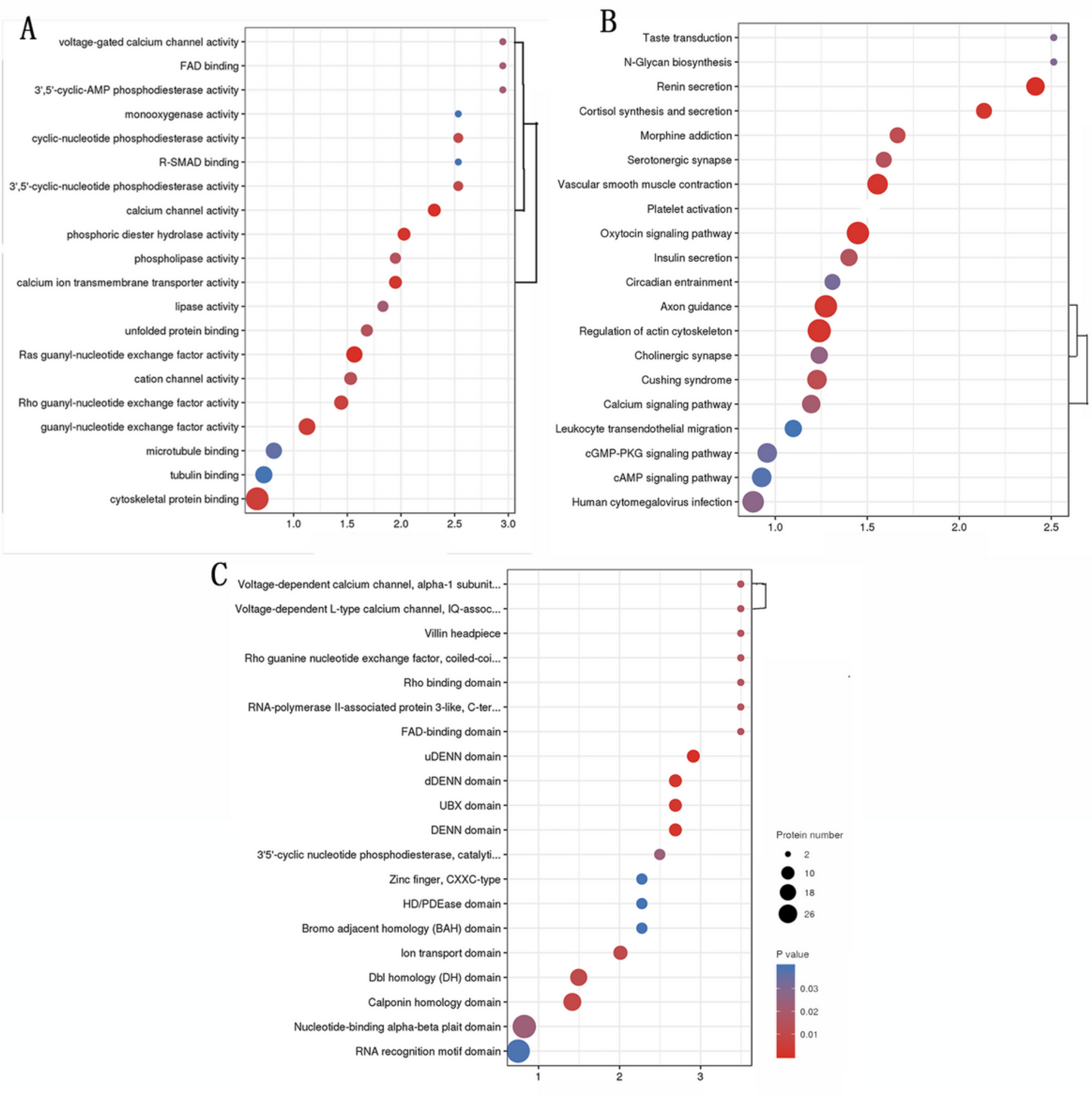

Figure 5

Bubble chart of enrichment and distribution of differentially modified proteins in AD cell models after OC1 administration(n=3). A, GO functional classification enrichment distribution bubble chart. B, KEGG pathway enrichment distribution bubble chart. $\mathrm{C}$, protein domain enrichment distribution bubble chart 


\section{A KEGG pathway}

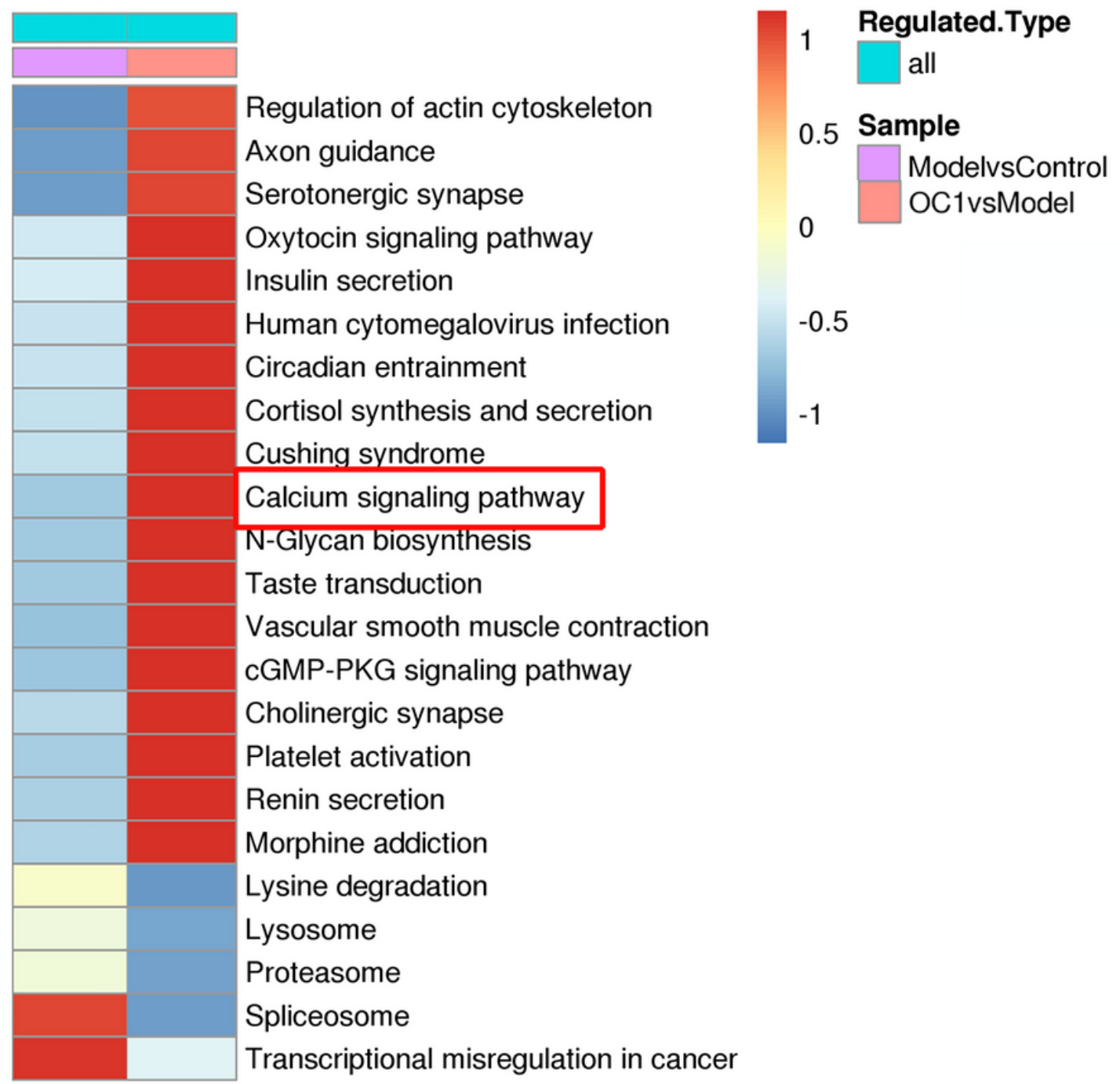

B

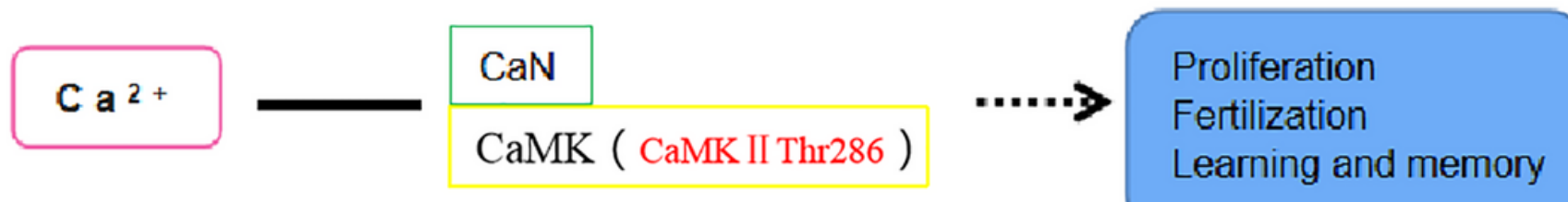

\section{Figure 6}

Cluster analysis of differentially modified proteins in AD cell model after OC1 administration. A, Heat map of cluster analysis of differentially modified proteins after OC1 administration( $n=3)$. B, Upstream and downstream relationship of CaMK》 protein in calcium signaling pathway $(n=3)$ 

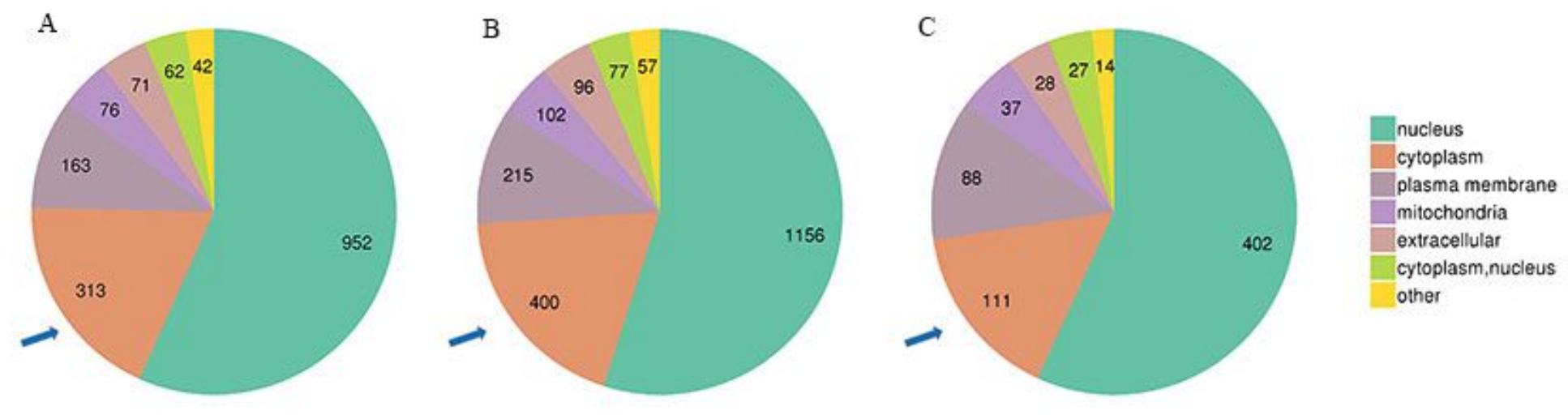

Figure 7

Statistical distribution map of the corresponding phosphorylated modification sites corresponding to CaMKII(Thr286) and Syn1(Ser603) in GO secondary classification. A, Model vs Control group. B, OC1 vs Control group. C, OC1 vs Model group
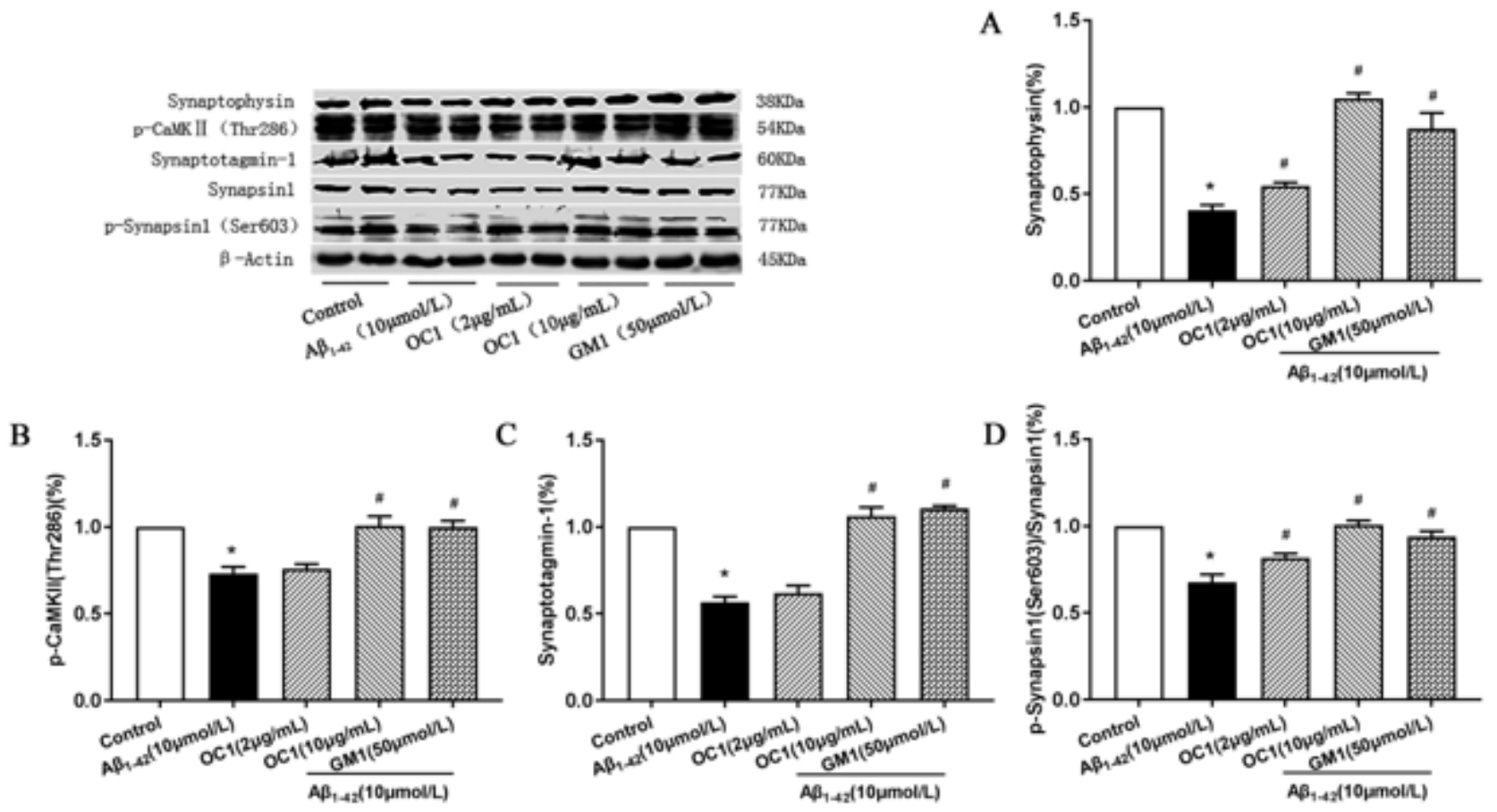

\section{Figure 8}

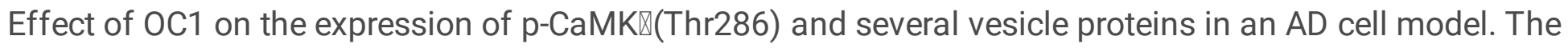
data are presented as the means \pm standard deviation (SD) of three individual experiments. A-D, Synaptophysin, p-CaMK®(Thr286), Synaptotagmin-1, p-Synapsin1(Ser603)/Synapsin1 protein expression in $A D$ cell model was examined by western blot and normailized to that of actin. ${ }^{*} \mathrm{P}<0.05$; compared with $A \beta 1-42$ group, \# $P<0.05$.

\section{Supplementary Files}


This is a list of supplementary files associated with this preprint. Click to download.

- Supplementarylnformation.pdf 